\title{
E-Therapy as a Therapeutic Strategy against Adolescent Obesity
}

\author{
Pedro Sousa ${ }^{1,2}$ \\ ${ }^{1}$ School of Health Sciences, Polytechnic Institute of Leiria, Leiria, Portugal \\ ${ }^{2}$ Health Research Unit (UIS) of the Polytechnic Institute of Leiria, Portugal \\ pedro.sousa@ipleiria.pt
}

The obesity has been recognized as the epidemic of the 21 st century. The obesity prevalence has increased to triple over the last twenty years.It is estimated that one child in every five in the European Region and one in three U.S. children suffer from overweight. To counter this trend requires the involvement of all health professionals in order to empower young people to maintain a healthy energy balance, to identify their problems/needs and to use rationally the available resources.

Advances in informatics, telecommunications and network technology have led to the emergence of a new revolutionary paradigm in healthcare that commonly is known as e-health. The technological opportunity, the need to establish a more efficient, economic and safehealth data sharing, and the evidence-based decision-making process requires a coordinated effort to bring out the power of etechnologies for health care.

The rapid growth of e-health is changing the way healthcare is provided. The development of the internet enables consumers to participate more actively in the decisions about their health care and assume more responsibility for managing their own care.

The information revolution continues to challenge health care professionals to think creatively about promoting health. It is estimated that a third of teenagers who use the internet look for health information, diets or exercise, which infers that the e-therapeutic interventions are not only appropriatebut maybe it's a real requirement for this population.

On the other hand, it is important to stress that, although the investigation focus the diet, exercise and behavioral strategies as the nuclear components of effective obesity treatment programs, its impact in the majority of the population is limited. Children today are growing up in a notoriously different context. Throughtechnologies,they are more intertwined than any previous generation. It is increasingly clear that ICT is and will continue to be even increasingly an integral part of the lives of children and adolescents, so that the teenagers ' behavioral change can have a longer range and be more effective if we use the channels that teens prefer and in formats that they recognize and will use.

Em Portugal foi recentemente desenvolvido um estudo pioneiro (Next.Stepproject) que conduziu ao desenvolvimento de uma comunidade e-terapêutica para adolescentes com excesso de peso e sua família.

These innovations are designed to bring health care to where they are really needed, providing the continuity between the clinical contexts and the daily life of the patients. The technology cannot replace medicine, but it may improve the effectiveness of standard clinical protocols and promote healthy lifestyles.

In Portugalit was recently developed a pioneering study (Next.Step project) which led to the development of ane-therapeuticcommunity for overweight teens and their family.The program includes a diverse set of resources, such as, educational resources (videos, brochures, menus, weekly tips, access to other links), self-monitoring (diet diaries, weight and physical activity records), social support (chats, discussion forums and personalized messages), interactive training modules (selfassessment quizzes, healthy recipes) and motivational tools (personal goals planning, treatment progression registry, positive reinforcement). The program has the direct support of an interdisciplinary team (including paediatrician, nutritionist, exercise physiologist and psychologist) who intervene whenever requested by the case manager.

This project assumed that the provision of a better treatment requires a more extensive contact with the clinical team and the use of alternative communication channels and interactive technologies with 
the adolescents and their families. The program was associated with an improvement of the positive perspective of life and of the health responsibility. These findings support the effectiveness of internet-based weight management programs involving overweight adolescents, as an add-on of the standard intervention.

This line of research and intervention is promising and may contribute to the inclusion of ICT in the treatment of overweight adolescents, through building technological interfaces that enable tailoring the intervention and streamline the monitoring.

Is this the future of pediatric healthcare?

We don't have a crystal ball but our vision for the future is clear: we want to deliver the most effective services and solutions to our children and families. 